\title{
SiPM technology applied to radiation sensor development
}

\author{
F. Risigo ${ }^{\mathrm{a}, *}$, A. Bulgheroni ${ }^{\mathrm{b}}$, M. Caccia ${ }^{\mathrm{a}}$, C. Cappellini ${ }^{\mathrm{a}}$, V. Chmill ${ }^{\mathrm{a}}$, N. Fedyushkina ${ }^{\mathrm{c}}$, A. Golubev $^{\mathrm{c}}$,

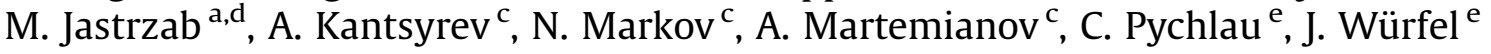 \\ a Physics and Mathematics Department, University of Insubria, via Valleggio 11, Como 22100, Italy \\ ${ }^{\mathrm{b}}$ Istituto Nazionale Fisica Nucleare, INFN sez. Milano, via Celoria 16, 20133 Milano, Italy \\ ' ITEP, Institute of Theoretica and Experimental Physics, B. Cheremushkinskaya 25, 117259 Moscow, Russia \\ ${ }^{\mathrm{d}}$ Electronics Department, AGH-University of Science and Technology, al. Mickiewicza 30, Krakow 30059, Poland \\ e PTW-Freiburg, Physikalisch-Technische Werkstaetten Dr. Pychlau Gmb, Loerracher Str. 7, 79115 Freiburg, Germany
}

\section{A R T I C L E I N F O}

Available online 1 April 2009

Keywords:

Silicon Photo-Multipliers

Radon monitoring

\begin{abstract}
A B S T R A C T
The Silicon Photo-Multiplier (SiPM) being yet in its infancy, a full protocol for the sensor characterization has been developed and implemented at the Physics Department of Universita' dell'Insubria. Sensors from different producers have been analyzed and compared, in view of the integration in the instruments for radiation detection. Exemplary illustrations are reported here, together with the first results on real-time dosimetry in mammography.
\end{abstract}

(c) 2009 Elsevier B.V. All rights reserved.

\section{Introduction}

The Silicon Photo-Multipliers (SiPM) [1] consist of a high-density $\left(\sim 10^{3} \mathrm{~mm}^{2}\right)$ matrix of diodes with a common output. Each diode is operated in a limited Geiger-Müller regime [2], in order to achieve gain at the level of $10^{6}$. As a consequence, these detectors are sensitive to single photons (even at room temperature), feature a dynamic range well above 100 photons/burst and have reasonable photon detection efficiency (PDE). Moreover, the SiPM measures the light intensity simply by the number of fired diodes.

Compactness, robustness, low cost, low operating voltage and power consumption represent the numerous advantages over traditional photodetectors.

Various SiPMs from three producers (Hamamatsu ${ }^{1}$, SensL $^{2}$ and $\mathrm{CPTA}^{3}$ ) have been qualified and compared. As exemplary illustration, the main characteristics of the two sensors that have been used for the measurements described in Section 3 are reported in Table 1.

Fig. 1 shows a typical oscilloscope output and the correspondent spectrum of the integrated signal for the Hamamatsu SiPM: the individual peaks are clearly separated from each other, allowing a straightforward photon counting.

\section{SiPM characterization}

The SiPM characterization procedure implemented at Universita' dell'Insubria has been performed on existing devices with the main

\footnotetext{
* Corresponding author. Tel.: +39031238 6142; fax: +390312386258.

E-mail address: fabio.risigo@uninsubria.it (F. Risigo).

${ }^{1}$ http://www.sensl.com

2 http://www.hamamatsu.com

${ }^{3}$ http://www.zao-cpta.ru/
}

goal to define an exhaustive protocol and to produce a comparative study. The tests have investigated parameters such as dark count rate (DCR), optical crosstalk, gain, photo detection efficiency and their temperature dependence. The characterization of the detectors is a major task with application-specific key figures.

For instruments designed for the detection of low-intensity light pulses, the photon detection efficiency and the dark count rate are the major figures. The former is determined by the quantum efficiency, the fill factor and the avalanche triggering efficiency, obviously linking it to the dark count rate. The DCR results from avalanches initiated by thermally generated carriers; however, because of the optical crosstalk [3], more cells can be fired in a correlated way, making the technology and operational optimization non-trivial. As exemplary illustration, values of the PDE versus overvoltage are shown in Fig. 2 for the detectors used in mammography (Table 1) and tested with green light at $524 \mathrm{~nm}$.

The "staircase", measuring the DCR versus the discrimination threshold, is shown in Fig. 3, where levels corresponding to the peak amplitude of the single, double and triple cells are evident.

For instruments sampling a "continuous" light flux, where the time scale is defined with respect to the single-cell signal development (see Fig. 1 for a typical pulse shape), the key figure is essentially the linear dynamic range, where significant deviations may be expected as long as the probability to hit a cell during its recovery time is not negligible. For these applications, single-cell signal piling-up is not an issue as long as the proportionality with the input stimulus is preserved.

Tests, performed with a focus on the dosimeter for mammography, prove the linearity of the sensors under test till avalanche triggering frequencies in excess of the $\mathrm{GHz}$ level.

For every application (whether oriented to the detection of pulses or sampling a continuous flux), thermal stability is an asset. 

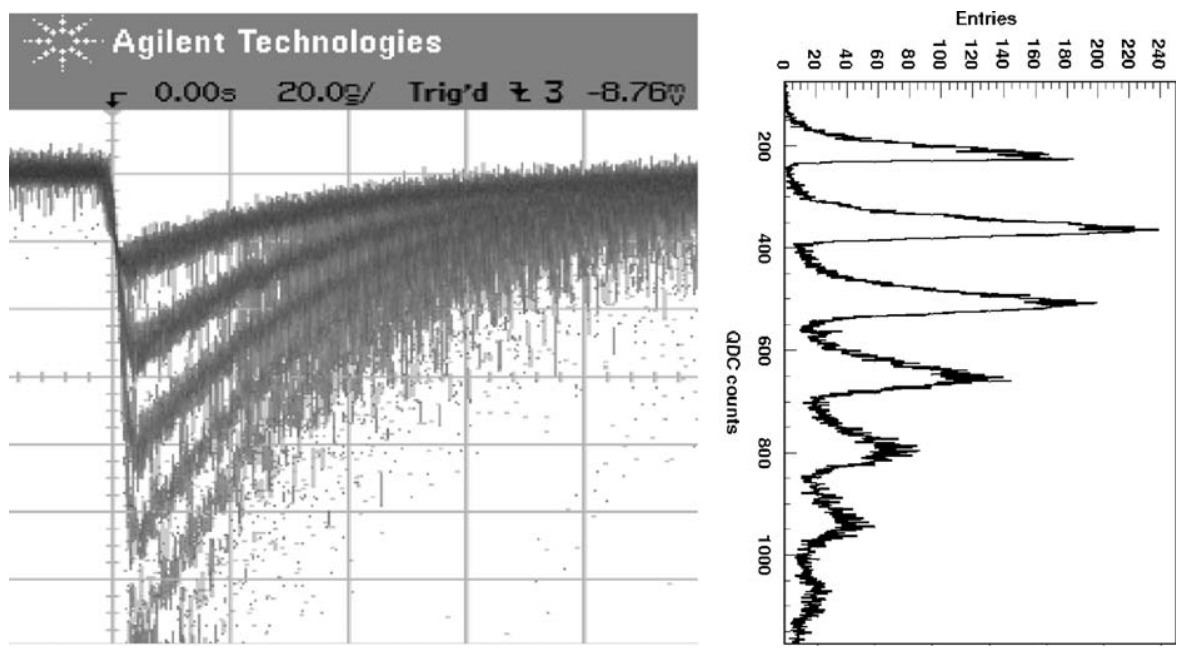

Fig. 1. Oscilloscope output and the correspondent multipeak spectrum for the Hamamatsu SiPM.

Table 1

Main characteristics of a SensL SiPM SMPScint1000 $\times 04$ and Hamamatsu SiPM MPPC S010361-050.

\begin{tabular}{lll}
\hline & SensL SiPM & Hamamatsu SiPM \\
\hline Number of diode & 8640 & 400 \\
Single-diode dimension & $20 \mu \mathrm{m} \times 20 \mu \mathrm{m}$ & $50 \mu \mathrm{m} \times 50 \mu \mathrm{m}$ \\
Breakdown voltage & $28.04 \mathrm{~V}$ & $70.56 \mathrm{~V}$ \\
Dark count rate & $8 \mathrm{MHz}$ & $270 \mathrm{kHz}$ \\
Optical crosstalk & $22.5 \%$ & $10 \%$ \\
Gain & $10^{6}$ & $7.5 \times 10^{5}$ \\
PDE & $4 \% \lambda=520 \mathrm{~nm})$ & $50 \%(\lambda=400 \mathrm{~nm})$ \\
\hline
\end{tabular}

Measurements refer to room temperature and a working overvoltage of $+2 \mathrm{~V}$.

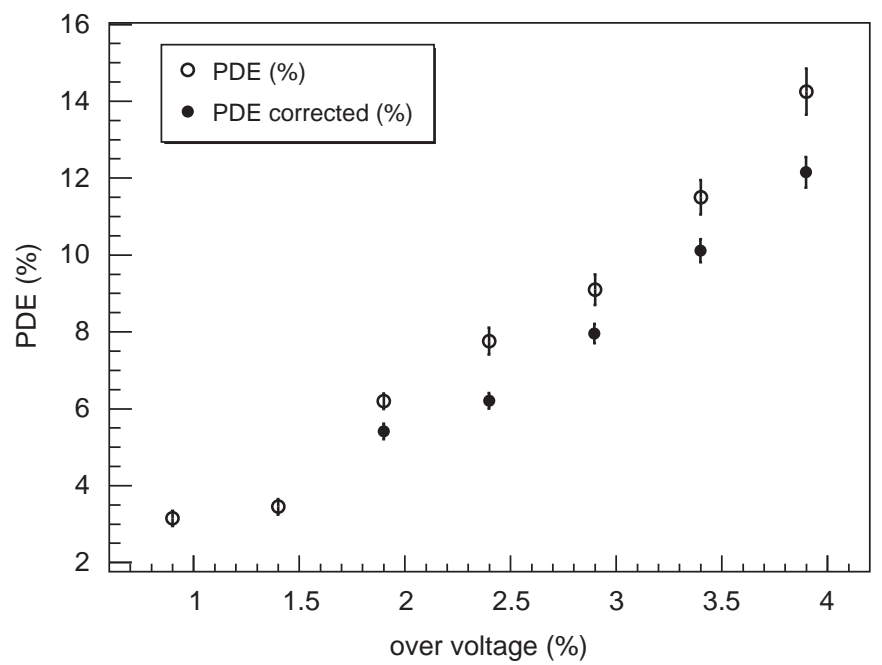

Fig. 2. PDE dependence versus overvoltage, for the Hamamatsu SiPM MPPC S010361-050; the plot shows the value for uncorrected PDE and corrected for the optical crosstalk.

Results do suggest that, apart from the obvious solutions of operating in a temperature-controlled environment, the possibility to implement a feedback procedure on the bias voltage is a viable option. Detailed measurements will be the subject of a forthcoming publication.

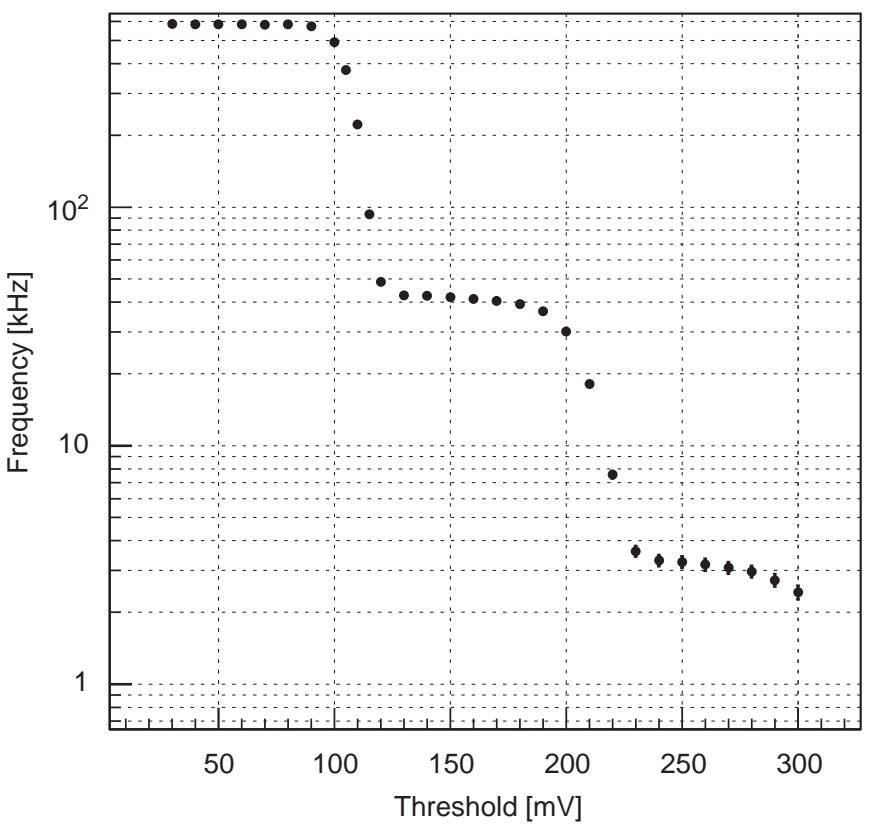

Fig. 3. DCR versus threshold for the Hamamatsu SiPM.

\section{Real-time dosimetry in mammography}

The real-time dose measurement in mammography is an exemplary application of the SiPM sensors.

This instrument is meant to be used during every mammography scan to monitor the dose delivered to the patient. It consists of a sensitive volume of scintillating, a Silicon PhotoMultiplier light sensor and a small local display of the recorded dose.

Two prototypes were characterized at PTW-Freiburg:

- Blue light-emitting plastic tile, optically coupled to a wavelength shifting fiber, in order to match the SiPM peak sensitivity.

- A green-emitting scintillating fiber, coupled to a clear fiber conveying the light to a SiPM. 
Table 2

Characterization results for the mammography device.

\begin{tabular}{|c|c|c|c|c|c|c|c|}
\hline \multirow[t]{2}{*}{ Set-up } & \multirow[t]{2}{*}{ Precision (\%) } & \multirow[t]{2}{*}{ Gain (QDC/mGy/s) } & \multirow[t]{2}{*}{ Sensitivity (mGy/s) } & \multirow[t]{2}{*}{ MDS (mGy/s) } & \multicolumn{3}{|c|}{ Max. dose rate (mGy/s) } \\
\hline & & & & & $<5 \%$ & $<7.5 \%$ & $<10 \%$ \\
\hline Tile-SensL & $1.95 \pm 0.05$ & $0.00500 \pm 0.00001$ & $0.45 \pm 0.01$ & $2.378 \pm 0.05$ & 40 & - & - \\
\hline Fiber-SensL & $3.17 \pm 0.05$ & $0.03286 \pm 0.00001$ & $2.67 \pm 0.04$ & $0.723 \pm 0.007$ & 115 & 155 & 190 \\
\hline Fiber-Hama & $2.07 \pm 0.03$ & $0.01691 \pm 0.00001$ & $1.82 \pm 0.02$ & $0.216 \pm 0.002$ & 170 & 200 & - \\
\hline
\end{tabular}

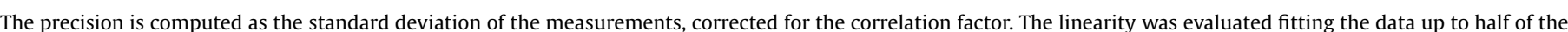

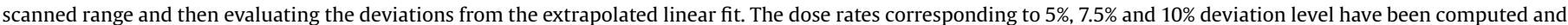
retained as indicators.

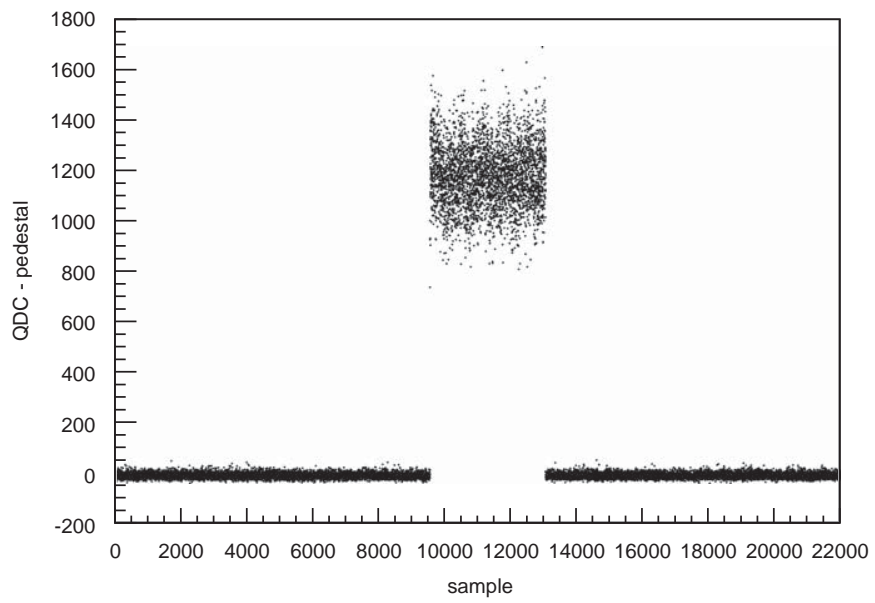

Fig. 4. The signal for a typical data set is shown as a function of the sample number. The integration time for the reported data was $3000 \mathrm{~ns}$ for a dose rate of $21 \mathrm{mGy} / \mathrm{s}$.

For the tile only the SensL SiPM sensor was used while the fiber system was also interfaced to the multi-pixel photon counter produced by HAMAMATSU (see Table 2 ).

The prototypes were exposed to the X-ray beam of a reference mammography unit and dose rates spanning from less than $1 \mathrm{mGy} / \mathrm{s}$ to a few hundreds $\mathrm{mGy} / \mathrm{s}$ have been used to irradiate the device in realistic conditions.

The charge from the sensor has been integrated and sampled at $30 \mathrm{kHz}$ by a QDC (charge digitizer), a transimpedance amplification stage (the QDC conversion capacitance is $0.11 \mathrm{pC}$ per QDC unit). Typical integration times were ranging between 500 and $5000 \mathrm{~ns}$ while the irradiation times were of $\sim 0.1 \mathrm{~s}$. For each irradiation, the effective dose rate and exposure time have simultaneously been measured with calibrated sensors.

The typical output signal from the prototype is displayed in Fig. 4, where the samples corresponding to the tile irradiation are clearly distinguishable.
The characterization of the prototype has been performed relying on four main figures of merit:

- precision, defined as the standard deviation of the measurements for constant radiation conditions;

- sensitivity, namely the minimum detectable variation in the dose rate;

- minimum detectable signal (MDS), differing from the sensitivity since it accounts as well for the spread in the offset and

- linearity, measured against the dose rate in the region of interest.

The system response to increasing dose rates has been measured and compared with the values provided by the UNIDOS. ${ }^{4}$ The precision and the linearity have been evaluated from these sets of measurements using both the tile and the fiber set-up.

A hint of the system performances, for the three configurations, is given in Table 2 while a complete report of the measurements procedure and results can be found in [4].

\section{Conclusion and outlook}

The working prototype described here has been designed and characterized during a research project funded by the European Commission within the sixth framework program. ${ }^{5}$ The project is successfully entering its last phase, and by the end of it all the working prototypes will be completely qualified.

\section{References}

[1] A. Adikinov, et al., Nucl. Instr. and Meth. A 387 (1997) 231.

[2] K.G. McKay, Phys. Rev. 94 (1954) 877.

[3] F. Zappa, S. Cova, M. Ghioni, A. Lacaita, C. Samori, Appl. Opt. 35 (12) (1996) 1956.

[4] C. Cappellini, et al., The RAPSODI project: SiPM development for applied research in radiation protection, in: 2008 NSS-MIC Conference Record.

\footnotetext{
${ }^{4}$ The UNIDOS, an ultrahigh-sensitive electrometer for charge and current measurement, produced by PTW-Freiburg.

5 Contract number: COOP 32993 - RAPSODI (RAdiation Protection with Silicon Optoelectronic Devices and Instruments).
} 